\title{
Correction to: The ASCRS Textbook of Colon and Rectal Surgery: Second Edition
}

David E. Beck

\section{Correction to:}

D.E. Beck et al. (eds.), The ASCRS Textbook of Colon and Rectal Surgery:

Second Edition, https://doi.org/10.1007/978-1-4419-1584-9

This book was inadvertently published with wrong copyright holder information. The original book has been updated accordingly.

Correct Copyright holder has been updated in the entire book: ASCRS (American Society of Colon and Rectal Surgeons).

The updated online version of this book can be found at https://doi.org/10.1007/978-1-4419-1584-9 